\title{
GROWTH MODEL WITH MIGRATION: STRUCTURE OF OPTIMAL SAVING RATES
}

\author{
ROBERT KRUSZEWSKI \\ Department of Mathematical Economics, Warsaw School of Economics \\ Al. Niepodlegtości 162, 02-554 Warszawa, Poland \\ E-mail:rkrusz@sgh.waw.pl
}

1. Introduction. The migration of persons is one of mechanisms for change in an economy's population and labour supply. The migration process, which means labour mobility, is analogous to capital mobility. Capital tends to move from places with a low rate of return to those with high rates of return. Labour tends to move from regions with low wage rates to regions with high wage rates.

Migration has a set of characteristics which are not like the characteristics of the natural population growth, e.g., the investments in new born members of the society are much more significant while the imigrant enters the society with a cluster of skills. First, migration contributes to the enlargement of the population size of the destination economy and represents corresponding losses for the source economy. Thus, we have to consider immigration and emigration as two sides of a single process. Second, unlike newly born persons, migrants come with accumulated human capital. Migrants typically do not carry much physical capital. Newborn children also differ from immigrants in that the residents of the economy tend to care of their children but not of immigrants. This difference in assimilation with the existing population implies differences in the way that population growth interacts with saving behaviour and, hence with rates of economic growth.

A starting point for the study of migration and growth is the Mankiw-Romer-Weil [2] model, which assumes a closed economy and an exogenous, constant saving rate. The extension to incorporate migration implies that economies open to some extent. The migration process also implies some degree of mobility of physical and human capital. We extend the above model to incorporate the influences of migration. Modified equations of motion for physical and human capital are derived.

Every household optimises discounted consumption (by a time-preference factor) over an infinite planning horizon, subject to the constraints given by the new equations of

2000 Mathematics Subject Classification: 49J15, 34H05, 34A30.

The paper is in final form and no version of it will be published elsewhere. 
motion for the human and physical capital. Solution of this dynamic optimization gives us a structure of optimal saving rates for both types of capital. Then, we determine how migration process affects the optimal saving rates.

2. The model. Let $M(t)$ be the flow of migrants into the domestic economy and $\bar{\kappa}$, $\bar{\lambda}$ be the quantity of physical and human capital, respectively, that each migrant brings along. In this model, the migrants cannot maintain any financial claims on foreign-source income. People who move, consume all the capital which they cannot carry with them. The domestic population and labour force, $L(t)$ grow at a constant rate $n$. The overall growth rate of the domestic population is therefore:

$$
\frac{\dot{L}}{L}=n+\frac{M}{L}=n+m
$$

where $m=\frac{M}{L}$ is the net migration rate.

The production function $Y(t)=F(K, H, L)$ has three inputs: physical capital $K(t)>$ 0 , human capital $H(t)>0$ and labour $L(t)>0$. The production function fulfils the neoclassical properties and Inada conditions. As in the Mankiw-Romer-Weil model we assume constant and exogenous technological progress $A(t)$, with a growth rate $x>0$ :

$$
\dot{A}(t)=x A(t) \text {. }
$$

The production function is homogeneous of degree one (constant returns to scale), so we get

$$
Y(t)=A(t) L(t) F\left(\frac{K(t)}{A(t) L(t)}, \frac{H(t)}{A(t) L(t)}, 1\right) .
$$

Let

$$
k \equiv \frac{K(t)}{A(t) L(t)}, \quad h \equiv \frac{H(t)}{A(t) L(t)}, \quad f(k, h)=\frac{F(K(t), H(t),(A(t) L(t))}{A(t) L(t)},
$$

then the production function can be written as:

$$
Y(t)=A(t) L(t) f(k, h),
$$

where $f(k, h)$ is the intensive form of the production function.

We assume that a fraction $s_{K}(t) \in(0,1)$ of output is invested:

$$
\dot{K}(t)=s_{K}(t) Y(t) .
$$

We assume that capital depreciates at a constant rate $\delta_{K}>0$. The change in the domestic capital stock is given by:

$$
\dot{K}(t)=s_{K}(t) Y(t)-\delta_{K} K(t)+\bar{\kappa} M(t) .
$$

The accumulation of human capital is modelled in the same way as physical capital, so the change in the domestic stock of human capital is given by:

$$
\dot{H}(t)=s_{H}(t) Y(t)-\delta_{H} H(t)+\bar{\lambda} M(t),
$$

where $s_{H}(t) \in(0,1)$ is a fraction of the output invested in accumulation of human capital, $\delta_{H}$ constant depreciation rate of human capital, $\bar{\lambda}$ quantity of capital that each migrant brings along. 
Equations (1), (3), (4) form a complete model of economic growth with migration. This is an extension of the Mankiw-Romer-Weil model, which incorporates the migration process.

To achieve analytical results in the next section we assume a Cobb-Douglas production:

$$
Y(t)=F(K, H, A L)=K^{\alpha} H^{\beta}(A L)^{1-\alpha-\beta}, \quad \alpha, \beta, \alpha+\beta \in(0,1),
$$

with Harrod neutral innovations (labour augmenting technological progress).

Rewriting the model in per effective unit of labour, we get the following equations of motion of the physical and human capital:

$$
\begin{aligned}
& \dot{k}=s_{K}(t) k^{\alpha} h^{\beta}-\left(\delta_{K}+x+n\right) k-m(k-\kappa), \\
& \dot{h}=s_{H}(t) k^{\alpha} h^{\beta}-\left(\delta_{H}+x+n\right) h-m(h-\lambda),
\end{aligned}
$$

where $\kappa=\frac{\bar{\kappa}}{A(t)}$ and $\lambda=\frac{\bar{\lambda}}{A(t)}$ are the amounts of physical and human capital per effective migrant, respectively, which are assumed to be constant.

The per effective unit of labour growth rates are:

$$
\begin{aligned}
& \gamma_{k}=s_{K}(t) k^{\alpha-1} h^{\beta}-\left(\delta_{K}+x+n\right)-m(1-\kappa / k), \\
& \gamma_{h}=s_{H}(t) k^{\alpha} h^{\beta-1}-\left(\delta_{H}+x+n\right)-m(1-\lambda / h) .
\end{aligned}
$$

The term $\delta_{K}+x+n$ is the effective depreciation rate for capital in the Mankiw-Romer-Weil model without migration. In this model, the effective depreciation rate is augmented by the migration term $m[1-\kappa / k]$. If $m=0$ or $\kappa=k$ in all points in time $t$, the result is identical as in the model without migration.

If $m<0$, then we compare the capital taken by emigrants with the capital of workers of the domestic economy. If $m>0$, then we compare capital brought in by immigrants with the capital of workers of the domestic economy.

The assumption $\kappa<k$ implies that a term $m[1-\kappa / k]$ increases the effective depreciation rate if $m>0$ and decreases if $m<0$.

The term $\delta_{H}+x+n$ is the effective depreciation rate for human capital in the Mankiw-Romer-Weil model without migration. In this model, the effective depreciation rate is augmented by the migration term $m[1-\kappa / k]$. If $m=0$ or $\lambda=h$ in all points in time $t$, the result is identical as in the model without migration.

If $m<0$, then we compare the human capital of emigrants with the human capital of workers of the domestic economy. If $m>0$, then we compare the the human capital of immigrants with the capital of workers of the domestic economy.

We neglect the possibility that a change in $k$ alters the selection of immigrants with respect to their capital $\kappa$. Moreover, if we assume that the typical economy is close to its steady state position, then we can treat $\kappa$ as constant over time. Hence, if $m>0$ then the ratio $\kappa / k$ would decline as $k$ rises. If $m<0$ then $\kappa$ represents the capital of each emigrant and in this case we can treat the ratio $\kappa / k$ as a constant.

The assumptions about human capital are the same as in the case of physical capital, so for $m>0$ the quotient $\lambda / h$ would decline as $h$ rises and stay constant for $m<0$. 
3. Optimal saving rates. The properties of the extended Mankiw-Romer-Weil model with constant exogenous saving rates have been analysed in [1]. The households had no influence on the level of investment in both types of capital and therefore on the level of consumption. Now this limitation will be removed. The saving rates change over time and the households choose the level of investments so as to maximize their discounted consumption in the infinite horizon.

In our case the overall consumption $C(t)$ is equal $\left(1-s_{K}-s_{H}\right) F(K, H, A L)$ and in per effective unit of labour

$$
c=\frac{C}{A L}=\frac{\left(1-s_{K}-s_{H}\right) A L f(k, h)}{A L}=\left(1-s_{K}-s_{H}\right) f(k, h)=\left(1-s_{K}-s_{H}\right) k^{\alpha} h^{\beta} .
$$

Homogeneous household maximizes its discounted consumption, which is equivalent to maximization of the functional $J(c(t))$ :

$$
J(c(t))=\int_{0}^{+\infty}\left(1-s_{K}(t)-s_{H}(t)\right) k^{\alpha} h^{\beta} e^{-\rho t} d t,
$$

where $s_{K}(t), s_{H}(t),\left(s_{K}(t)+s_{H}(t)\right) \in(0,1)$ for all $t \in \mathbb{R}_{+}$. The term $e^{-\rho t}$ is a discount factor and $\rho>0$ is a discount rate (time preference rate). The higher value of discount rate means that households prefer today's consumption to tomorrow's consumption.

Consumption possibilities of a typical household in the considered model are restricted by the equations of motion. The equations of motion describe the evolution over time the per effective unit of labour of human and physical capital:

$$
\begin{aligned}
& \dot{k}=s_{K}(t) k^{\alpha} h^{\beta}-\left(\delta_{K}+x+n\right) k-m(k-\kappa), \\
& \dot{h}=s_{H}(t) k^{\alpha} h^{\beta}-\left(\delta_{H}+x+n\right) h-m(h-\lambda),
\end{aligned}
$$

which we derived above.

The way to determine the optimal time paths of saving rates in physical and human capital equals to the solution of the following optimal control problem:

$$
\begin{gathered}
\text { maximize } J(c(t))=\int_{0}^{+\infty}\left(1-s_{K}(t)-s_{H}(t)\right) k^{\alpha} h^{\beta} e^{-\rho t} d t \\
\text { subject to } \dot{k}=s_{K}(t) k^{\alpha} h^{\beta}-\left(\delta_{K}+x+n\right) k-m(k-\kappa), \\
\dot{h}=s_{H}(t) k^{\alpha} h^{\beta}-\left(\delta_{H}+x+n\right) h-m(h-\lambda), \\
k(0)=k_{0}, \quad h(0)=h_{0},
\end{gathered}
$$

where the saving rates are control variables, while the amount of human and physical capital are state variables. This optimal control problem can be solved by the Maximum Principle of Pontryagin. To do this we set up a current-value-Hamiltonian:

$$
\begin{gathered}
H\left(s_{K}(t), s_{H}(t), k, h, \nu_{K}, \nu_{H}\right)=\left(1-s_{K}(t)-s_{H}(t)\right) k^{\alpha} h^{\beta}+\nu_{K}\left(s_{K}(t) k^{\alpha} h^{\beta}\right. \\
\left.-\left(\delta_{K}+x+n\right) k-m(k-\kappa)\right)+\nu_{H}\left(s_{H}(t) k^{\alpha} h^{\beta}-\left(\delta_{H}+x+n\right) h-m(h-\lambda)\right),
\end{gathered}
$$

where $\nu_{K}(t), \nu_{H}(t)$ are co-state variables associated with $k$ and $h$ respectively. First order necessary conditions for maximization of the functional $J(c(t))$ subject to given 
constraints are:

$$
\begin{gathered}
\frac{\partial H}{\partial s_{K}}=0, \\
\frac{\partial H}{\partial s_{H}}=0, \\
\dot{\nu}_{K}=-\frac{\partial H}{\partial k}+\nu_{K} \rho, \\
\dot{\nu}_{H}=-\frac{\partial H}{\partial h}+\nu_{H} \rho,
\end{gathered}
$$

and the transversality conditions

$$
\begin{aligned}
& \lim _{t \rightarrow+\infty} \nu_{K} e^{-\rho t} k(t)=0, \\
& \lim _{t \rightarrow+\infty} \nu_{H} e^{-\rho t} h(t)=0 .
\end{aligned}
$$

Necessary conditions for the current-value-Hamiltonian take the following form:

$$
\begin{gathered}
-k^{\alpha} h^{\beta}+\nu_{K} k^{\alpha} h^{\beta}=0 \\
-k^{\alpha} h^{\beta}+\nu_{H} k^{\alpha} h^{\beta}=0 \\
\dot{\nu}_{K}=-\alpha\left(\left(1-s_{K}-s_{H}\right) k^{\alpha-1} h^{\beta}-\nu_{K} \alpha s_{K} k^{\alpha-1} h^{\beta}-\nu_{H} \alpha s_{H} k^{\alpha-1} h^{\beta}\right. \\
+\left(\delta_{K}+x+n\right)+m+\nu_{K} \rho, \\
\dot{\nu}_{H}=-\beta\left(\left(1-s_{K}-s_{H}\right) k^{\alpha} h^{\beta-1}-\nu_{K} \beta s_{K} k^{\alpha} h^{\beta-1}-\nu_{H} \beta s_{H} k^{\alpha} h^{\beta-1}\right. \\
+\left(\delta_{H}+x+n\right)+m+\nu_{H} \rho, \\
\dot{k}=s_{K}(t) k^{\alpha} h^{\beta}-\left(\delta_{K}+x+n\right) k-m(k-\kappa), \\
\dot{h}=s_{H}(t) k^{\alpha} h^{\beta}-\left(\delta_{H}+x+n\right) h-m(h-\lambda) .
\end{gathered}
$$

From (9) and (10) we get $\nu_{K}(t)=1$ and $\nu_{H}(t)=1$ for all $t \in \mathbb{R}_{+}$. Hence

$$
\begin{aligned}
& \lim _{t \rightarrow+\infty} \nu_{K}(t) e^{-\rho t} k(t)=\lim _{t \rightarrow+\infty} k(t) e^{-\rho t}=0, \\
& \lim _{t \rightarrow+\infty} \nu_{H}(t) e^{-\rho t} h(t)=\lim _{t \rightarrow+\infty} h(t) e^{-\rho t}=0,
\end{aligned}
$$

so the transversality conditions are fulfilled. Substituting

$$
\begin{gathered}
\nu_{K}(t)=\nu_{H}(t)=1, \\
\dot{\nu}_{K}(t)=\dot{\nu}_{H}(t)=0
\end{gathered}
$$

into equations (11), (12) we get the following system of equations:

$$
\left\{\begin{array}{l}
\alpha k^{\alpha-1} h^{\beta}=\delta_{K}+x+n+m+\rho, \\
\beta k^{\alpha} h^{\beta-1}=\delta_{H}+x+n+m+\rho .
\end{array}\right.
$$

If we differentiate logarithms of the above equations with respect to time then we get

$$
\left\{\begin{array}{l}
(\alpha-1) \frac{\dot{k}}{k}+\beta \frac{\dot{h}}{h}=0 \\
\alpha \frac{\dot{k}}{k}+(\beta-1) \frac{\dot{h}}{h}=0
\end{array}\right.
$$


From (16) we get that the growth rates of physical and human capital are zero under the assumption that the households behave rationally. Dividing (13) and (14) by $k$ and $h$, respectively, and considering the zero growth rates of both types of capital we obtain:

$$
\begin{aligned}
& s_{K} k^{\alpha-1} h^{\beta}-\left(\delta_{K}+x+n\right)-m(1-\kappa / k)=0, \\
& s_{H} k^{\alpha} h^{\beta-1}-\left(\delta_{H}+x+n\right)-m(1-\lambda / h)=0 .
\end{aligned}
$$

Substituting to those equations the quantities

$$
\begin{aligned}
k^{\alpha-1} h^{\beta} & =\frac{1}{\alpha}\left(\delta_{K}+x+n+m+\rho\right), \\
k^{\alpha} h^{\beta-1} & =\frac{1}{\beta}\left(\delta_{H}+x+n+m+\rho\right),
\end{aligned}
$$

derived from (15) we get the following formulas for optimal saving rates in our model:

$$
\begin{aligned}
& s_{K}=\alpha \frac{\delta_{K}+x+n+m(1-\kappa / k)}{\delta_{K}+x+n+m+\rho}, \\
& s_{H}=\beta \frac{\delta_{H}+x+n+m(1-\lambda / h)}{\delta_{H}+x+n+m+\rho} .
\end{aligned}
$$

To get the final structure of optimal saving rates we need to determine the optimal values of $k$ and $h$. Let $\mu=\delta_{K}+x+n+m+\rho, \eta=\delta_{H}+x+n+m+\rho$ then from (15) we have

$$
\begin{aligned}
& k=\left(\alpha^{1-\beta} \beta^{\beta} \mu^{\beta-1} \eta^{-\beta}\right)^{\frac{1}{1-\alpha-\beta}}, \\
& h=\left(\alpha^{\alpha} \beta^{1-\alpha} \mu^{-\alpha} \eta^{\alpha-1}\right)^{\frac{1}{1-\alpha-\beta}} .
\end{aligned}
$$

The optimal values of physical and human capital, derived from first order conditions are constant and completely determined by the parameters describing the economy. Without loss of generality of our considerations, let us assume that $\delta_{K}=\delta_{H}=\delta$ and $\gamma=$ $\delta+x+n+m+\rho$. This assumption simplifies the algebra of further calculations. Hence, the optimal saving rates are constant and equal:

$$
\begin{aligned}
& s_{K}=\alpha \gamma^{-1}\left(\delta+x+n+m\left(1-\kappa\left(\alpha^{1-\beta} \beta^{\beta} \gamma^{-1}\right)^{\frac{-1}{1-\alpha-\beta}}\right)\right), \\
& s_{H}=\beta \gamma^{-1}\left(\delta+x+n+m\left(1-\lambda\left(\alpha^{\alpha} \beta^{1-\beta} \gamma^{-1}\right)^{\frac{-1}{1-\alpha-\beta}}\right)\right) .
\end{aligned}
$$

Following our assumptions, $\alpha, \beta,(\alpha+\beta) \in(0,1)$ we conclude that

$$
\begin{aligned}
& \gamma^{-1}\left(\delta+x+n+m\left(1-\kappa\left(\alpha^{1-\beta} \beta^{\beta} \gamma^{-1}\right)^{\frac{-1}{1-\alpha-\beta}}\right)\right) \in(0,1), \\
& \gamma^{-1}\left(\delta+x+n+m\left(1-\lambda\left(\alpha^{\alpha} \beta^{1-\beta} \gamma^{-1}\right)^{\frac{-1}{1-\alpha-\beta}}\right)\right) \in(0,1) .
\end{aligned}
$$

So that $s_{K}, s_{H},\left(s_{K}+s_{H}\right) \in(0,1)$ for all $t \in \mathbb{R}_{+}$. The derived saving rates $s_{K}$ and $s_{H}$ satisfy all necessary conditions to maximize the functional $J(c(t))$ subject to the constraints (13) and (14). Hence, there exists a solution to the problem of maximization of the household's discounted consumption stream.

To assess the model's quantitative implications, we need a rough estimate of the parameters. We follow Romer [3] and assume that: $\alpha=0.35, \beta=0.4, x+n+\delta=0.1$. Additionally we assume that $\kappa=3, \lambda=4$ and $\rho=0.02$ as a low and $\rho=0.15$ as high values of a time preference factor. 
Now we will show the influence of migration on the optimal saving rates. If the time preference factor is low then for a small rate of immigration both saving rates increase (figure 1).
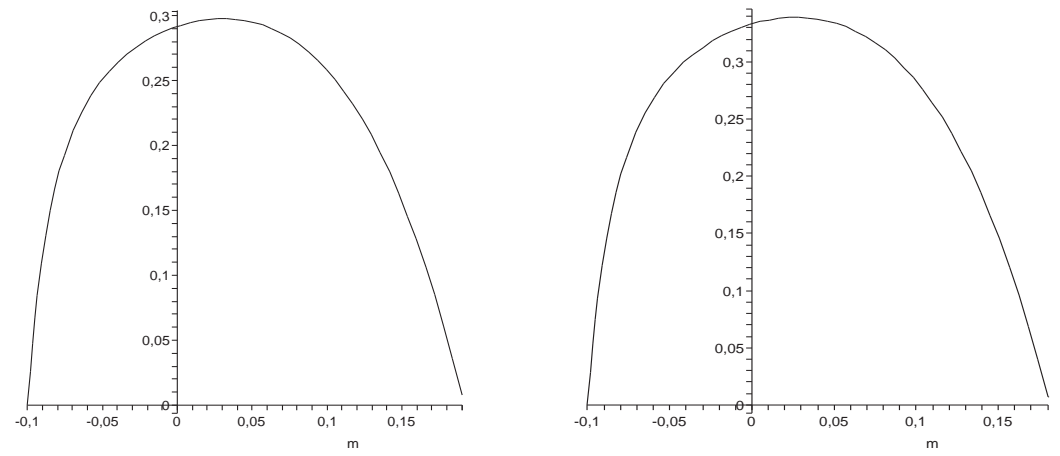

Fig. 1. Optimal saving rates as a function of migration rate $s_{K}$ (left) and $s_{H}$ (right). Low values of time preference factor.

If the immigration rate is too high then the optimal saving rates go down to zero. When there is a low inflow of immigrants, rationally behaving households will decrease their today's consumption which in turns means higher saving rates, but the overall consumption in the infinite horizon will be greater due to the higher output which is a result of immigration. When the time preference factor is high the behaviour of households will be different (figure 2).
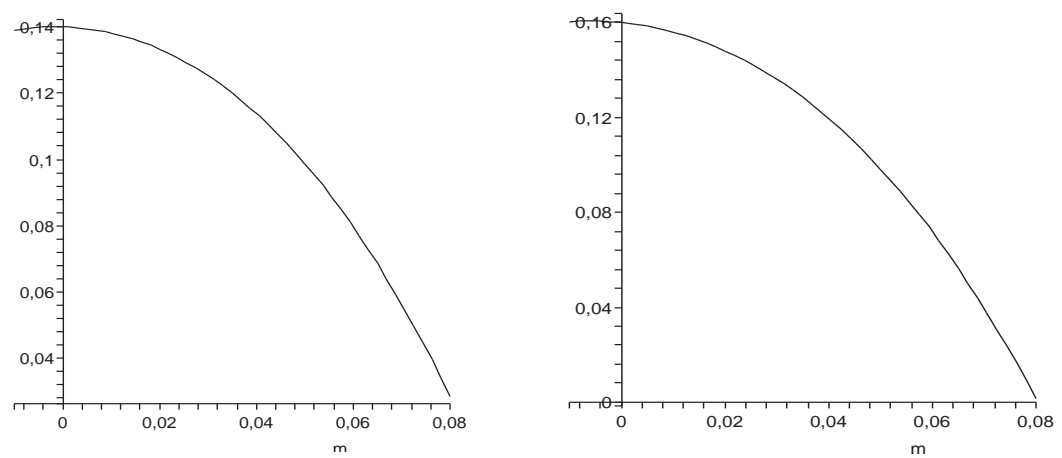

Fig. 2. Optimal saving rates as a function of migration rate $s_{K}$ (left) and $s_{H}$ (right). High values of time preference factor.

It means that the optimal saving rates go down to zero and investments hold little incentive for household as its growth is not optimal.

\section{References}

[1] R. Kruszewski, Dynamics of the economic growth model with migration, Discussion Papers, Series Mathematical Economics, No. 1/EM/2003. 
[2] N. G. Mankiw, D. Romer and D. N. Weil, A contribution to the empirics of economic growth, Quarterly Journal of Economics 107 (1992), 407-437.

[3] D. Romer, Advanced Macroeconomics, McGraw-Hill 1996.

[4] T. Tokarski, Determinants of economic growth in a case of constant returns to scale, Department of Economics, University of Łódź, 2001. 